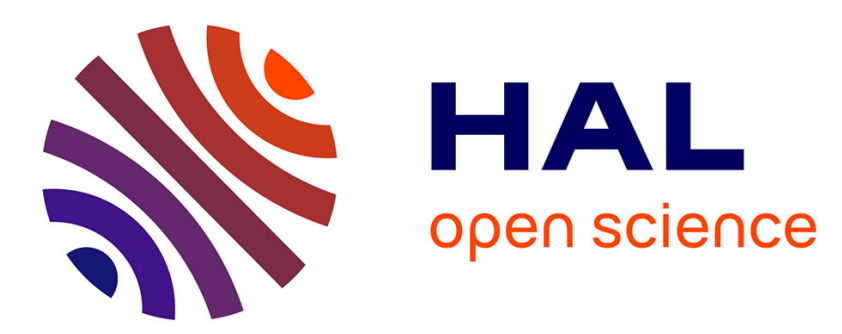

\title{
Expérience, trajectoire d'apprentissage et processus d'émancipation dans un dispositif de formation par la pratique théâtrale
}

Catherine Archieri

\section{- To cite this version:}

Catherine Archieri. Expérience, trajectoire d'apprentissage et processus d'émancipation dans un dispositif de formation par la pratique théâtrale. Travail et apprentissages: revue de didactique professionnelle, 2014, 12, pp.34. hal-01068094

\section{HAL Id: hal-01068094 \\ https://hal.science/hal-01068094}

Submitted on 24 Sep 2014

HAL is a multi-disciplinary open access archive for the deposit and dissemination of scientific research documents, whether they are published or not. The documents may come from teaching and research institutions in France or abroad, or from public or private research centers.
L'archive ouverte pluridisciplinaire HAL, est destinée au dépôt et à la diffusion de documents scientifiques de niveau recherche, publiés ou non, émanant des établissements d'enseignement et de recherche français ou étrangers, des laboratoires publics ou privés. 
Titre : Expérience, trajectoire d'apprentissage et processus d'émancipation dans un dispositif de formation par la pratique théâtrale.

Résumé : De nombreux dispositifs de formation d'adultes revendiquent, parallèlement à la visée d'intégration ou d'adaptation professionnelles, l'ambition d'encourager le développement d'individus lucides et autonomes. L'interrogation se porte alors sur les catégories de situations à proposer aux apprenants pour favoriser cette visée émancipatrice : émancipation vis à vis de quel référentiel ? Quels liens avec la pratique professionnelle cible ? Quel format dans un curriculum?

C'est dans un dispositif de formation utilisant la pratique théâtrale - plus précisément le jeu dramatique -, conforme à cette option, que nous avons analysé l'expérience en train de se faire et le processus d'apprentissage de jeunes adultes se destinant au métier d'enseignant.

Cet article présente l'activité d'une étudiante en combinant différents niveaux d'analyse, d'un point de vue temporel et social (Lemke, 2000). A travers le croisement d'analyses synchronique (analysant les moments particuliers d'apprentissage) et diachronique (analysant l'évolution de ces apprentissages sur une durée de plusieurs séances) les résultats mettent en évidence, d'une part, les phénomènes et processus sous-jacents participant aux transformations - notamment silencieuses - par l'activité du jeu dramatique, d'autre part, comment se construit l'expérience en relation avec les autres formés, les formateurs, le chercheur et enfin illustrent les liens qui se tissent entre les différentes expériences vécues et leurs incidences sur le développement personnel.

Mots-clés : formation professionnelle ; jeu dramatique ; trajectoire d'apprentissage ; construction identitaire ; autonomie ; émancipation.

Title: Experience, learning trajectory and process of emancipation in a training program using the practice of drama.

\begin{abstract}
Many adult training programs, along with the purpose of integration or professional adaptation, claim to encourage the development of lucid and autonoumous individuals. The question is then focused on the types of situations to provide learnees in order to promote this emancipatory project: emancipation against what standard? Which links with the targeted professional practice? What format in a curriculum?

It is in a training program using the practice of drama - specifically dramatic play - complying with this option, that we have analyzed the experience being done and the learning process of young adults intending to become teachers.

This paper presents the activities of a student by combining different levels of analysis, in a temporal and social point of view (Lemke, 2000). Through a synchronic (analyzing the specific moments of learning) and diachronic (analyzing the evolution of the learning over a period of several sessions) cross analysis, the results show, on the one hand, phenomena and underlying processes contributing to the - including silent - transformations induced by the activity of acting, on the other hand, how the experience is built in relationship with others trainees,
\end{abstract}


trainers, researcher and, finally, illustrate the links being forged between the different experiences and their impact on the personal development.

Keywords : vocational training, drama acting, learning trajectory, identity construction; autonomy emancipation.

\section{Introduction}

L'objet de nos travaux de recherche se centre sur la relation entre le travail et la formation. Notre intérêt est de savoir si former selon un environnement déconnecté de l'activité cible peut répondre aux exigences professionnelles d'un métier, celui d'enseignant en l'occurrence. Dans le cadre de cet article, la focale portera sur les incidences d'une pratique théâtrale sur la construction de la dynamique identitaire des formés, sur la corrélation éventuelle entre construction identitaire et émancipation. Nous employons l'expression construction identitaire dans l'acception qu'en donnent Barbier (1996), Bourgeois (1998) et Kaddouri (2000) : le choix d'utiliser le terme de dynamique identitaire permet de signifier que l'identité n'est pas une donnée figée, constituée une fois pour toute. Elle est à considérer dans son évolution elle n'est pas qu'un «étant» mais également un « devenir ». (Kaddouri, 2000).

Le terme émancipation ${ }^{1}$, sera utilisé au sens de s'affranchir de représentations diverses, issues de l'environnement au sens large et indéternimé.

Dans le cadre de cette étude, il s'agit pour nous de décrire l'expérience d'une étudiante dans un contexte de formation par le jeu dramatique, pratique théâtrale reconnue a priori bénéfique pour l'agir en classe (Page, 2001).

L'objet d'intérêt social est le suivant: quelles constructions d'expérience et, de fait, quels apprentissage/développement ${ }^{2}$ sont possibles par le jeu dramatique ? Autrement dit de façon pragmatique : a-t-on quelque chose à gagner à se confronter au jeu dramatique pour se préparer au métier d'enseignant ?

\section{Cadre théorique}

Nous avons fait le choix d'une approche « analyse de l'activité humaine » pour mener cette étude. Ce choix est légitimé par le développement d'outils théoriques et méthodologiques de compréhension de l'activité humaine par le courant de l'ergonomie de langue française et de la psychologie du travail. Ce choix correspond à notre volonté de rendre intelligible la construction de l'organisation et la signification de l'activité de l'apprenant.

\footnotetext{
${ }^{1}$ Du latin "emancipare", affranchir un esclave du droit de vente, venant de "e" privatif et "manucapare", prendre par la main (L'achat des esclaves se faisait en les prenant par la main). L'émancipation est un acte juridique qui soustrait, de manière anticipée, un mineur à la puissance parentale ou à sa tutelle afin de le rendre capable d'accomplir tous les actes de la vie civile nécessitant la majorité légale : gérer ses biens, percevoir ses revenus, réaliser des actes d'administration... En disposant de sa pleine capacité juridique, le mineur est assimilé à un majeur.

Par extension, dans le langage courant, émanciper signifie affranchir d'une autorité, d'une domination, d'une tutelle, d'une servitude, d'une aliénation, d'une entrave, d'une contrainte morale ou intellectuelle, d'un préjugé...

${ }^{2}$ Par apprentissage nous entendons l'acquisition, par les individus et les collectifs qu'ils constituent, de modes d'action nouveaux pour eux, issus du patrimoine culturel (...) en relation ou non avec un projet intentionnel de transmission de ces modes d'action par une instance de formation; par développement nous entendons la transformation du répertoire d'actions des individus et des collectifs qu'ils constituent, en relation ou non avec un projet intentionnel d'accompagnement par une instance de formation (Durand, 2008).
} 
Notre approche de l'activité humaine s'inscrit dans le courant des approches « orientées activités » reposant sur le postulat de l'enaction (Varela, 1989). De fait nous utilisons l'approche du « cours d'action » (Theureau, 2004, 2006) qui permet de décrire et comprendre les formes d'interactions entre l'acteur et son environnement. Il s'agit alors d'identifier les organisateurs et les régularités de l'activité en situation. Pour comprendre les modalités d'adaptation individuelle, nous accordons le primat à la signification que donne l'acteur de son vécu immédiat. Celui-ci est alors considéré comme une construction et une articulation progressives de significations qui se déploient au fil du déroulement de l'action (Theureau, 2009).

Cette recherche en intelligibilité est en relation avec l'intention d'aider à la conception ergonomique de situations de formation (Guérin, 2012).

En effet, nos travaux s'inscrivent dans la lignée de ceux menés par Leblanc, Ria, Dieumegard, Serres, et Durand (2008) sur l'analyse de l'activité dans une approche enactive pour concevoir des dispositifs de formation professionnelle des enseignants. A cet égard, « la formation est envisagée comme une construction empirique et scientifique, évolutive et adaptative, étroitement articulée à une analyse de l'activité ». (Leblanc \& all, 2008).

\section{Objet d'étude}

Cette étude croise deux niveaux d'analyse : une synchronique (analyse d'un moment particulier du dispositif de formation), l'autre diachronique (analyse de l'évolution des apprentissages sur la durée du dispositif) afin de rendre compte de la construction de l'expérience et son incidence sur le développement d'une étudiante ${ }^{3}$.

La question de recherche relative à la perspective synchronique est la suivante : quels sont les éléments de l'environnement de la situation théâtrale qui participent à la construction de significations et organisent l'action de l'étudiante au cours d'un épisode inaugural du dispositif de formation?

Concernant la perspective diachronique, l'enjeu sera de pister la transformation des composantes de l'activité, l'engagement de l'étudiante au fil d'épisodes de jeu dramatique identifiés dans le déroulement chronologique du dispositif de formation. La question de recherche dans cette perspective diachronique est : quelles sont les transformations repérables sur la durée de la formation, relatives à la construction du sens pour l'étudiante, de sa pratique théâtrale?

\section{Terrain d'étude}

Nous avons suivi le cas exemplaire de Clara ${ }^{4}$, volontaire pour participer à cette étude, étudiante en M2 STAPS 5 . Agée de 26 ans, Clara s'est engagée de ce dispositif ${ }^{6}$ de formation avec l'attente de gagner de assurance pour affronter les oraux d'admissibilité du CAPEPS ${ }^{7}$. Le dispositif « atelier théâtre » a été proposé à titre facultatif à des étudiants de Master $1^{\text {ère }}$ et $2^{\text {ème }}$ année «métiers de l'enseignement $»^{8}$ site de Brest de l'IUFM de Bretagne 9 .

\footnotetext{
${ }^{3}$ Cette étude est extraite d'un programme de recherche plus large, consacré à la construction de l'expérience par le théâtre, programme qui a fait l'objet d'une thèse de doctorat soutenue le 8 juillet 2014, consultable à partir du lien suivant : http://hal.univ-brest.fr/tel-00865641/

${ }^{4}$ L'usage d'un pseudonyme a fait partie du contrat établi avec l'étudiante pour garantir son anonymat.

${ }^{5}$ Sciences et Techniques des Activités Physiques et Sportives.

${ }^{6}$ Le terme «dispositif» est utilisé dans l'acception définie par Albero (2010) : en formation, l'emploi de ce terme «met en valeur l'interrelation entre l'offre et l'usage et la mise en adéquation des formes d'intervention prévues par les concepteurs avec les comportements effectifs des publics destinataires ».

${ }^{7}$ Certificat d'Aptitude au Professorat d'Education Physique et Sportive.

${ }^{8}$ Professeurs des écoles (PE) et professeurs en lycées et collèges (PLC).

${ }^{9}$ L'IUFM de Bretagne est l'actuel ESPE de Bretagne.
} 
Il a été conçu et animé de façon conjointe par deux formateurs d'origine professionnelle différente: une comédienne et un formateur IUFM. Le dispositif est composé de six séances sur un empan temporel d'un mois et demi.

C'est un dispositif conçu comme un «Espace d'Action Encouragée » (EAE), selon la définition qu'en donne Durand (2008) qui, à la suite de différents auteurs (Peirce, 1995 ; Varela, 1996 ; Lahire, 1998 ; Bourdieu, 1998) en définit le fondement essentiel : la possibilité d'émergence de « dispositions à agir ». Ce dispositif a été bâti à la suite d'un observatoire de l'activité d'étudiants d'une promotion antérieure ${ }^{10}$. Concernant les composantes interprétatives, émotionnelles, cognitives de l'activité, les dispositions à agir visées relèvent du social, de l'individuel et s'actualisent comme émergentes, réglées, régulières ou inédites selon les situations rencontrées et selon le moment de l'histoire de chaque individu dans le dispositif d'activité encouragée.

\section{Recueil des matériaux empiriques}

Le dispositif de formation a été présenté aux participants comme associé à un observatoire ${ }^{11}$ de recherche, fondé sur des entretiens d'autoconfrontation réalisés à partir de traces vidéo recueillies en situation de formation. Le principe des entretiens d'autoconfrontation est de permettre l'accès au niveau pré-réflexif ${ }^{12}$ de la conscience de l'acteur. Le niveau pré-réflexif de l'activité offre l'intérêt d'être à la fois significatif du point de vue de l'acteur et de pouvoir donner lieu à des descriptions et explications valides et utiles pour le chercheur, sans prétendre pour autant rendre compte de l'ensemble des niveaux d'organisation de l'activité.

L'action et les communications de Clara pendant le dispositif «atelier théâtre » ont été enregistrées à l'aide d'une caméra numérique posée sur un pied en dehors du plateau et des micros H.F. fixés au plafond. Un plan large a permis de filmer en continu son action ainsi que celles des autres personnes situées dans son environnement proche. À la fin de chaque journée et parfois à la pause méridienne, Clara a participé à des entretiens d'autoconfrontation. À partir des traces vidéo, elle a été invitée par le chercheur à commenter les images de ses propres prestations à exprimer son ressenti, à auto-estimer son état de confort / inconfort. Pour ce faire, l'échelle d'estimation des états affectifs (échelle EEA) a été mobilisée (Ria, L., Sève, C., Durand, M., Bertone, S., (2004) $)^{13}$. Au travers d'un questionnement centré sur le vécu immédiat de Clara en situation de jeu dramatique, le chercheur a cherché à accéder à la construction de sens et notamment à ses préoccupations, ses focalisations, ses interprétations.

\section{Choix des objets théoriques mobilisés}

\footnotetext{
${ }^{10}$ Ce point est développé dans un article paru dans la revue Recherche \& formation sous le titre « Pratique du jeu dramatique et construction de l'expérience » (Guérin, G. \& Archieri, C, 2013).

${ }^{11}$ La notion d'observatoire, évoquée dans les travaux de Theureau $(2004,2006)$ qualifie l'ensemble des outils méthodologiques adoptés dans les enquêtes empiriques et insiste sur la notion de «construction » des données : les données n'existent pas en soi mais sont produites par le questionnement théorique et empirique du chercheur. ${ }^{12}$ Le postulat de conscience pré-réflexive caractérise une modalité particulière de vécu, constitutive de l'activité. Il s'agit de la familiarité de l'acteur à lui-même qui accompagne le flux de son activité (l'action de lire s'accompagne de la conscience de lire mais ne constitue pas une action nouvelle distincte de celle de lire). Cette présence de l'acteur à son activité se caractérise comme son « effet de surface ».

${ }^{13}$ Cette échelle permet à l'acteur d'estimer et d'exprimer synthétiquement le caractère positif ou négatif de son expérience. Elle est constituée de sept points : de +3 (très agréable ou très confortable) à -3 (très désagréable ou très inconfortable). L'estimation sur cette échelle de la tonalité de ses états affectifs correspond à l'intensité de l'expérience émotionnelle, à un ressenti syncrétique et immédiat. Il s'agit du niveau montrable (et non racontable) des émotions. L'estimation par l'acteur du caractère positif ou négatif de son expérience se déroule en visionnant la vidéo. L'étudiant peut à tout instant modifier les valeurs estimées sur l'échelle EEA sans avoir à les justifier.
} 
Les verbalisations provoquées et les enregistrements vidéo ont ensuite été analysés en référence au cadre sémiologique du cours d'action (Theureau, 2006) selon deux niveaux. Le premier, correspondant à l'approche synchronique, a été réalisé en mobilisant le « cours d'expérience », objet théorique qui permet de rendre compte de la dynamique de construction de l'expérience lors de la pratique de jeux dramatiques. Le second, relatif à l'approche diachronique, a été de rendre compte des transformations de l'activité sur la durée de la formation. L'objet théorique utilisé a été le « cours de vie relatif à une pratique ».

\section{Pourquoi le jeu dramatique ? Définition et focus sur deux situations de jeu}

Les situations de jeu relatives à la pratique du jeu dramatique ont été retenues comme des espaces privilégiés d'observation pour pister la trajectoire de développement de l'étudiante.

A cela, plusieurs raisons : a) ces situations partagent le même contexte d'une prise de parole individuelle face à un collectif, prise de parole emblématique de la pratique professionnelle cible, b) ces situations de formation répondent à la plupart des attentes exprimées par les étudiants en début du dispositif de formation, c) ces situations font partie des épisodes les plus renseignés au cours des sessions d'autoconfrontation auxquelles ont participé les étudiants volontaires.

A la suite de Ryngaert (1991), nous énumérons ainsi les caractéristiques du jeu dramatique : a) le jeu dramatique ne vise pas une reproduction exacte de la réalité, b) le jeu dramatique est une activité collective. Le groupe est le lieu où l'individu s'élabore pour soi et pour les autres, devant les autres et pour les autres, c) le jeu dramatique n'est pas subordonné au texte. Celui-ci est remplacé par une parole improvisée ou établie à partir d'un canevas, c) le jeu dramatique ne réclame pas des acteurs virtuoses rompus à toutes les techniques d'expression théâtrales. Ce n'est ni la perfection du geste ni celle de l'imitation qui est recherchée, mais un comportement lucidement élaboré à l'intérieur d'une situation.

Pratique théâtrale mais aussi activité éducative, le jeu dramatique est présenté par les théoriciens du théâtre comme propre à favoriser le développement personnel des individus, «dans le sens d'une éducation à la liberté ». (Page, 2004).

Notre choix s'est porté sur deux situations de jeu: l'épisode inaugural du dispositif en matière de jeu dramatique : l'exercice «je ne veux pas boire de café ». C'était un exercice à réaliser seul face au public composé des pairs. Il s'agissait d'entrer sur le plateau pour venir adresser un message simple : « je ne veux pas boire de café ». La contrainte était de respecter quatre temps : 1) Entrer sur le plateau avec une tasse de café à la main, 2) Poser la tasse sur le sol,

3) Adresser le message au public : « je ne veux pas boire de café », 4) Sortir du plateau.

Il était précisé par la formatrice, en présentation de la tâche à réaliser, qu'il ne fallait pas chercher à "signifier » quoique ce soit, à «jouer un rôle ». Ce qui serait commenté, c'est ce que le corps allait exprimer par le rythme du déplacement, la circulation du regard sur le groupe, la tonalité de la voix, l'utilisation des temps de silence, par exemple.

Le second épisode traité a eu lieu le dernier jour du dispositif de formation. Il s'intitulait : «l'exercice d'improvisation libre». Il s'est déroulé sur une durée imposée de trois minutes et chronométrée au cours desquelles le joueur devait parler en s'adressant au public composé de pairs, sans longue pause, sans contrainte 
particulière de chercher à raconter quoi que ce soit, de signifier quelque chose. «Juste parler pendant la durée, sans s'arrêter, sans s'enfermer en soi-même, mais en adressant son propos » a annoncé la formatrice.

Ces deux situations ont beaucoup marqué Clara qui a demandé à en voir les traces vidéo et y a consacré du temps ${ }^{14}$ pendant les entretiens d'autoconfrontation. Les tâches réalisées au cours de ces situations ont été choisies dans le cadre de cet article parce qu'elles relèvent de couplages types ainsi que nous avons pu le constater à travers les résultats des études menées auprès de plusieurs autres étudiants impliqués dans l'observatoire ${ }^{15}$.

\section{Traitement des données}

Les extraits d'entretiens d'autoconfrontation et les traces vidéo relatifs à ces deux épisodes ont fait l'objet d'un traitement sous forme de récit réduit aux unités significatives élémentaires ${ }^{16}$ à partir desquelles on a proposé une analyse locale ${ }^{17}$ de l'activité de Clara. Par l'association de ces descriptions, les analyses locales ont consisté à caractériser les éléments signifiants de l'environnement qui perturbent le couplage structurel entre le joueur et sa situation à l'instant « $\mathrm{t}$ » d'une situation de jeu. Ce traitement relève de l'analyse sémiologique développée dans les travaux de Theureau $(2004,2006,2009)$.

Les unités élémentaires, l'engagement et les préoccupations relatifs à ces deux épisodes ont été documentés par trois chercheurs impliqués dans le programme de recherche en ergonomie des situations de formation. Chaque point de désaccord a été discuté de manière à atteindre un taux d'agrément de $100 \%{ }^{18}$.

\section{Résultats}

\section{Episode « café » : une expérience autocentrée}

L'analyse locale dans la perspective synchronique réalisée sur l'épisode «je ne veux pas boire de café » laisse apparaître des phénomènes saillants de l'activité de l'étudiante que nous synthétisons ici ${ }^{19}$.

Clara s'engage dans l'activité de mauvaise grâce. L'expérience que les formateurs lui proposent de vivre ne correspond pas aux attentes qu'elle a eu l'occasion d'exprimer en début de formation ( «faire des exercices qui me permettront de gagner en assurance ») ni à ses représentations de la catégorie d'exercices qui pourrait lui permettre de prendre confiance en elle. De plus, elle redoute de se retrouver seule face au collectif, collectif dont elle ne connait qu'une partie (6 personnes sur 16 participants).

\footnotetext{
${ }^{14}$ La durée consacrée à ces deux situations de jeu en entretiens d'autoconfrontation a été respectivement d'1h10 (café) et $45 \mathrm{mn}$ (improvisation libre).

${ }^{15} \mathrm{Cf}$. rapport de thèse, http://hal.univ-brest.fr/tel-00865641/

${ }^{16}$ Elles correspondent à des actions, communications, interprétations, focalisations, émotions significatives pour l'acteur à l'instant considéré. Le flux de l'activité est appréhendé à partir de la succession de ces signes. Tout en considérant l'activité de l'acteur comme un continuum, la théorie sémiologique du cours d'action admet la nécessité d'établir des découpages afin de permettre l'extraction d'une ou plusieurs périodes du cours d'expérience de l'acteur.

${ }^{17}$ Le traitement par l'analyse locale consiste à découper chaque unité selon les catégories du signe hexadique (Theureau, 2004).

${ }^{18}$ Le format de cet article ne permet pas de développer les modalités de traitement des données. Pour en prendre connaissance, on pourra consulter l'article paru dans la revue Recherche \& développement sous le titre « Pratique du jeu dramatique et construction de l'expérience » (Guérin, G. \& Archieri, C, 2013).

${ }^{19}$ Le format de l'article nous empêche de produire le traitement des données issu de l'analyse locale par le signe hexadique. L'ensemble est développé en annexe de notre thèse de doctorat consultable à partir du lien suivant : http://hal.univ-brest.fr/tel-00865641/

Les citations produites dans le corps de l'article, qui ont servi à la construction des données, sont extraites des entretiens d'autoconfrontation menés avec Clara.
} 
Elle s'engage dans la situation de ce jeu dramatique qu'elle juge angoissante dont elle ne perçoit visiblement pas le sens («A quoi ça sert de venir dire ça devant les autres?»). La stratégie qu'elle met en œuvre consiste à retarder le moment de son passage pour se donner l'occasion d'observer la manière dont ses collègues s'emparent de la consigne. Pour cela, elle évite d'attirer l'attention des formateurs sur elle pour ne pas être désignée pour présenter sa prestation. Elle centre son attention sur les personnes qu'elle connait (les étudiants M2 STAPS) et juge inopportun de se focaliser sur les prestations des étudiants PE qu'elle ne se sent pas apte à analyser puisqu'elle ne connait pas ces étudiants dans la vie « réelle », donc n'est pas en mesure d'évaluer l'écart entre «le vrai et le semblant». En revanche, elle est surprise devant les prestations de ses collègues proches qu'elle qualifie de «surjouées » parce que déconnectées d'un comportement ordinaire. Clara se sent également en désaccord avec les commentaires développés par la formatrice à l'issue de chaque passage de jeu ; elle est désarçonnée de constater que les prescriptions de la formatrice évoluent au fil des prestations et se transforment même (EEA : -2). Son inconfort augmente encore au constat que certains participants sont invités à refaire leur prestation. Cette activité d'enquête qui vise à anticiper son passage n'aura pas pour Clara l'effet escompté de rassurance, bien au contraire. Elle est dans l'incapacité de planifier son activité, les informations qu'elle reçoit étant trop contradictoires entre ce qu'elle observe (ses amis qu'elle ne « reconnaît » pas), ce qu'elle entend (les commentaires de la formatrice qu'elle juge «mensongers ») et son inconfort consécutif («je me demandais ce que je faisais là, c'était atroce ») (EEA : -3). C'est pourquoi elle décide de suspendre cette phase d'observation qu'elle estime «stérile » et précipite sa prestation en se portant volontaire pour passer, cherchant à éviter de se retrouver la dernière à passer, cherchant à «se débarrasser» de la tâche à réaliser.

La prestation de Clara sur l'exercice « je ne veux pas boire de café » marque les quatre temps prescrits par la formatrice mais sa posture, faussement décontractée (elle s'appuie sur un pilier présent sur le plateau) trahit une tentative maladroite de masquer son inconfort. Les commentaires collectifs sur sa prestation exprimeront bien cet état de fait, chose qu'elle ne niera pas après sa prestation. Invitée à recommencer son parcours par le formateur, Clara refusera sans ambages (EEA : -1). Son engagement traduit la préoccupation de vouloir se « débarrasser de la tâche » en référence à des situations similaires d'inconfort : les oraux de concours, le fait de s'adresser à des inconnus. La posture corporelle d'appui contre le pilier sera largement commentée en entretien, Clara constatant par les images que le corps exprime l'inconfort qu'elle a cherché à masquer pendant l'action. Elle rappelle alors les propos préliminaires à l'exercice émis par la comédienne : le corps raconte une histoire et c'est ça qui est intéressant à percevoir, sans masquage.

Les micro-mondes (Varela, 1996) de Clara dans cette situation peuvent être qualifiés d'autocentrés : elle ne vise pas à répondre à la tâche prescrite mais à s'en débarrasser. Elle renonce à comprendre les attentes de la comédienne, à décoder, dans l'activité de ses collègues, des indices pour agir, à planifier son activité à venir. Elle interrompt soudain son observation pour se livrer, dans la précipitation, à l'expérimentation. Le temps de réalisation sera très court: 24 secondes. Elle se focalisera sur les quatre temps du scénario proposé en « oubliant» de s'adresser au public. Clara s'est coupée du collectif, ses préoccupations relèvent d'un masquage d'inconfort. Au moment des commentaires sur sa prestation, elle ne niera pas son état d'inconfort. Elle ne saisira pas l'opportunité de reproduire l'expérience parce l'amélioration de sa performance n'est pas une de ses préoccupations. A ce stade du dispositif, l'étudiante considère ce contexte de formation par le jeu dramatique comme un espace générateur d'inconfort, aux prescriptions paradoxales et perturbatrices. 


\section{Episode « improvisation libre » : une expérience d'ouverture sur l'environnement}

L’épisode de jeu dramatique « improvisation libre» se déroule le dernier jour du dispositif de formation, soit cinq séances après l'épisode « café ». Les participants de l'atelier théâtre se connaissent mieux, ils se côtoient en dehors du temps de plateau, lors des pauses méridiennes. Le collectif est maintenant organisé : les barrières entre filières d'étude PE et PLC sont tombées. Plusieurs situations de jeu dramatique ont été proposées aux joueurs, c'est à présent un espace de formation connu et reconnu.

L'exercice «improvisation libre » est le pendant d'une autre situation où les participants étaient invités à demeurer immobiles, en silence, pendant trois minutes, en interaction avec le public, juste par le regard. Clara évoquera à plusieurs reprises ses difficultés «à regarder un interlocuteur dans les yeux » au cours des entretiens d'autoconfrontation. Cette situation d'improvisation parlée relève de cette «contrainte » de regarder dans les yeux, prescription dont Clara dira être maintenant capable de s'accommoder "parce qu'elle est en confiance avec les autres » (EEA : 0).

Clara décrit le temps qui précède son passage comme consacré à l'observation des prestations des autres participants qui se succèdent et consacré également à la recherche du sujet qui fera l'objet de son monologue pendant trois minutes. Elle précise, en entretien d'autoconfrontation, avoir trouvé plaisant en situation le monologue de Laëtitia, une étudiante de M2 PLC EPS qui décrit une anecdote qui vient de se dérouler à la machine à café pendant la pause.

Clara sera la dernière à passer pour cet exercice. Elle s'engage dans l'activité, relativement détendue $(E E A:+2)$, en ayant planifié un seul point: la thématique qu'elle va développer («je vais parler de ce qui est arrivé ce matin, pendant le trajet en voiture, avec les copains, pour arriver jusqu'ici »). Consciente de la durée à tenir, elle passe par de nombreuses digressions (le temps qu'il lui faut le matin pour se préparer, son indécision pour choisir ses vêtements, ses retards perpétuels, les retrouvailles entre collègues, etc.). L'interaction avec le public fonctionne puisque ses propos provoquent des rires qui la rassurent et l'encouragent à poursuivre. Elle dit, en entretien, se laisser porter en situation par son état « ici et maintenant», dans l'interaction avec le public (EEA : +2). La durée à tenir n'est pas un problème pour l'étudiante qui ne cherche plus à se débarrasser de la tâche, comme dans l'épisode précédent, mais avoue qu'elle « aurait pu tenir beaucoup longtemps que les trois minutes chronométrées ». Elle s'adresse vraiment aux membres du public c'est-à-dire leur accorde des regards, ponctue son monologue de pauses oratoires, prend en compte leurs réactions.

Clara s'appuie dès lors sur un répertoire d'expériences vécues ${ }^{20}$, sur des régularités construites et qui peuvent être formulées ainsi :

- «L'improvisation en atelier théâtre relève du même processus de communication que la prise de parole dans un cercle familier »

- «Les autres participants de l'atelier ne me jugent pas »

- «Il faut que j'accepte l'état émotionnel que je ressens dans certaines situations de l'atelier, et que je fasse avec »

Clara a rompu avec ses habitudes d'action liées à l'anticipation et accepte de prendre en compte son état, sans masquage (« si le rire vient, je laisse venir »).

\footnotetext{
${ }^{20}$ Deux exercices de jeu dramatique ont été expérimentés dans le temps entre les séances évoquées pour cette étude.
} 
Au cours de ce deuxième épisode, on constate que l'apprenante a augmenté son territoire d'action : son réseau d'interactions interpersonnelles mais aussi l'espace et le temps de jeu. Elle saisit les opportunités que lui offre l'activité : les rires des pairs, les regards complices. Elle se «laisse porter», suit le fil de son inspiration et a la sensation confortable de répondre aux attentes supposées de la formatrice à travers cet exercice. Disponible, en confiance dans un environnement qu'elle juge propice et protégé, elle est en mesure de laisser émerger des possibles de jeu et développe notablement sa capacité à «adresser sa parole» à travers l'appui que constitue le regard.

Lorsqu'on interroge Clara sur la congruence entre les contenus de formation proposés en cette fin de dispositif et ses attentes initiales, l'étudiante explique qu'elle aurait aimé faire ce type d'exercice dès le début puis se reprend en reconnaissant qu'elle n'en aurait sans doute pas été capable.

A l'issue de cette analyse, on note la manifestation d'un processus de construction d'une forme typique : Clara a repéré un air de ressemblance entre différents moments de jeu et souligne l'intérêt dans la «vraie vie » de ce vécu en espace fictionnel et protégé de formation.

\section{Discussion}

La description de la dynamique de la construction de l'expérience de Clara rend compte de la trajectoire de développement vécue par cette étudiante ${ }^{21}$. Le croisement des approches synchronique et diachronique nous a permis de suivre une trajectoire d'apprentissage et d'en pister les éléments saillants. Cette expérience qui relie différentes composantes -émotionnelles, motrices et symboliques - semble spécifique aux apprenants débutants en théâtre (Archieri, 2013) ayant peu confiance en eux-mêmes et une grande anxiété à l'idée de ne pas disposer d'un répertoire de connaissances spécifiques - connaissances techniques ou attitudes - pour faire face à ce genre de situations. Nous aborderons trois points en discussion de cette étude empirique : la notion de transformations « silencieuses », celle de « dispositions à agir » et enfin les effets transformant des entretiens menés dans le cadre du protocole de recherche associé.

\section{Des transformations « silencieuses »}

Les résultats, issus de l'analyse des deux situations, illustrent une transformation notable de l'engagement de Clara entre le $1^{\text {er }}$ jour de formation et le $6^{\text {ème }}$ : l'intention de masquage d'inconfort est remplacée par l'acceptation de son état à l'instant « $\mathrm{t} »$. Nous faisons donc l'hypothèse que l'apprenante a réalisé des liens de signification entre les différentes expériences vécues, qu'elle a repéré des traits de similarité entre les exercices proposés, et a actualisé une « disposition à jouer » pour être en mesure de répondre à la tâche : nous nommerons cette disposition «le lâcher-prise ». Nous qualifierons cette disposition comme un acte de conscience libérateur qui permet de changer de comportement et de dépasser les habitudes d'actions limitantes et de l'orienter différemment. C'est une aptitude proche de ce qui peut être qualifié trivialement de «prendre confiance ». Ainsi, ce résultat nous permet d'affirmer, à la suite de Zeitler (2011) que tout changement d'habitude dans l'action constitutive de l'expérience génère la construction d'une confiance en soi dans un environnement spécifique.

\footnotetext{
${ }^{21}$ Le présent article est consacré au cas unique de Clara. Des résultats à partir d'un panel plus large d'étudiants sont disponibles à partir du lien suivant : http://hal.univ-brest.fr/tel-00865641/
} 
La capacité à entrer en interaction avec le collectif est à souligner également comme vecteur de développement chez Clara. Incapable de regarder les collègues n'étant pas issus de son cercle amical, elle précise en fin de protocole qu'elle est en voie de régler ce problème, dans d'autres contextes aussi que celui de la formation. La boucle courte, opérée au sein du dispositif de formation est en passe de se muer en boucle longue, c'est-à-dire dans d'autres environnements que celui protégé de la formation.

Les « dispositions à agir » ne sont pas contrôlées par un soi substantiel et unifié mais par une pluralité de «micro-identités » construites historiquement dans la répétition de "micro-mondes» (Varela, 1996). Contrairement à l'impression de continuité que nous avons dans l'attention ordinaire, l'activité cognitive n'est pas un processus ininterrompu mais une alternance de «micro-identités » et de «micro-mondes ». Les instants de prise de conscience (comme celui du développement des interactions collectives pour Clara) ne sont que des brèches dans l'attitude ordinaire de la vie quotidienne. Parce qu'elle est globale, opérant en amont, au stade de la maturation, la transformation est toujours « silencieuse » (Kaddouri, 2000). L'événement qui est le marqueur et l'indice de la transformation, lui, s'individualise. En l'occurrence pour le cas qui nous occupe, l'épisode du monologue au cours duquel Clara se sent être capable de regarder l'ensemble du groupe et le fait, est perçu par l'étudiante comme un temps fort de formation et de mise en sens de soi, à propos duquel ce qu'elle exprime relève de « la justesse du vivre ou de la conduite ». (Jullien, 2001, 2009).

\section{Des « dispositions à agir » à l'épreuve des résistances individuelles}

Nous avons eu l'occasion de le dire plus haut, cet artefact a été conçu selon le principe d'un espace d'actions encouragées (Durand, 2008) et donc se fonde sur des postulats identifiés qui sont : l'intention de congruence entre le projet des concepteurs et les attentes des participants, l'action de formation à effets différés dans un environnement cible, la possibilité de saisie d'opportunités. Nous développerons ici deux aspects de conception particulièrement saillants relativement au déroulé des épisodes étudiés que sont : a) le caractère paradoxal de la prescription: incomplétude, injonction à la subjectivité, transformation de la tâche au fil du déroulé des prestations des participants, étayage, etc., telles peuvent être caractérisées les consignes de jeu, b) le dépassement de la zone proximale de développement : l'enjeu est de faire faire aux acteurs ce qu'ils sont supposés ne pas savoir faire en les confrontant à de l'inattendu, de l'inconnu, pour les amener à incarner leurs émotions dans les espaces du jeu dramatique.

Il s'est avéré, in situ et à plusieurs reprises pendant les entretiens d'autoconfrontation, que Clara relève ces aspects de conception de l'environnement de formation comme cause d'inconfort pour elle. En effet, parmi les préoccupations exprimées relatives aux moments précédant l'entrée dans le jeu, l'étudiante pointe ses difficultés à saisir « la commande », voire l'enjeu même de la tâche prescrite par les formateurs. L'incitation à l'expression de l'affect et au non-masquage des émotions dans les jeux proposés a eu aussi pour effet de déstabiliser Clara, davantage sur la réserve propre aux espaces de formation classiques, plus axés sur les savoirs disciplinaires et leur transposition didactique. Nos résultats laissent apparaître une notion-clé que tout espace de formation basé sur la pratique du jeu dramatique devrait prendre en considération : les résistances, au sens psychanalytique du terme, ou système de défense par lequel l'individu se protège quand il se sent en danger, ou dans l'inconfort.

Ainsi, le recours systématique et ritualisé dans le dispositif à la parole de l'apprenant, pour exprimer son ressenti, semble être un point à plébisciter. De la même manière, la construction d'un espace partagé de coopération dont la signification serait partagée entre concepteurs et apprenants semble une piste à développer. Enfin, il est 
nécessaire, pour que les visées de développement personnel puissent être atteintes, d'inscrire ce type de dispositif dans la durée - l'exemple du dispositif en six séances étudié étant un minimum -, et sans doute aussi d'en conserver le caractère facultatif. En effet, il est important, dans ce type d'approche centrée sur la construction identitaire, de laisser le choix d'entrer dans un environnement qui nécessitera, à un moment ou à un autre, une démarche introspective.

\section{La place des entretiens d'autoconfrontation dans le curriculum}

Identifier les transformations sous-jacentes aux modifications de l'action suppose une collaboration avec les apprenants permettant d'analyser leur activité dans le dispositif en question. Cette approche compréhensive s'envisage sous différentes formes, entre autres l'analyse de pratiques (Altet, 2000), l'entretien d'explicitation (Vermersch, 1994, 2002), l'entretien d'autoconfrontation (Theureau, 2004) tel que nous l'avons envisagé pour cette étude empirique. C'est dans ce contexte que l'acteur peut, sous certaines conditions de collaboration, accéder à une partie de son activité non perçue en situation. En faisant raconter, montrer, mimer, simuler, commenter ce qui est significatif pour l'acteur dans une situation particulière, on accède à cette conscience préréflexive qui est une compréhension partielle par lui-même de son activité ; elle n'est pas ajoutée à, mais constitutive de son activité (Theureau, 2006). La mise à jour de ce couplage ou significations est une phase indispensable pour influencer l'activité des acteurs qui seuls définissent leurs situations, pour privilégier l'émancipation de chacun.

Cela nécessite de la part du formateur et des formés de développer les compétences pour investir de manière suffisamment fine cette analyse de l'activité réelle au travail et exploiter en formation ces nouvelles données. Cette dimension itérative est un idéal de formation, que l'association à un protocole de recherche rend possible. Pour notre part, nous avons fait le constat au cours de cette étude empirique des effets notables des entretiens d'autoconfrontation. Ils se sont avérés constituer une ressource précieuse pour l'étudiante : comme prolongation de l'activité pour continuer à comprendre et interpréter son action en dehors de l'espace de formation, comme sécurisation de l'expérience - notamment pour évacuer la pression émotionnelle -, comme support de conscientisation de l'apprentissage/développement. Nous avons pu noter l'évolution au fil des semaines du statut des entretiens auprès de Clara : de "prothèse » rassurante pour éviter le décrochage, les entretiens sont devenus « une aide», un vecteur d'intelligibilité, de conscientisation, voire un moteur de transformation pour l'étudiante.

Les différentes activités de Clara (explicitation du vécu, prises de conscience) dans la situation d'entretien d'autoconfrontation mettent en évidence que l'articulation entre le dispositif de formation et le protocole de recherche intervient à plusieurs niveaux et selon plusieurs logiques qui doivent être repérées. Les résultats ont d'ailleurs montré qu'en début de formation, des prises de conscience au cours des entretiens, donc en dehors des situations théâtrales, avaient joué un rôle important dans la poursuite de l'investissement de Clara dans la formation.

\section{Conclusion}

L'étude de cas développée dans cet article permet de tirer des enseignements car, bien de que type idiosyncrasique, elle est représentative de l'action de plusieurs étudiants ayant participé à l'observatoire plus large mis en place dans le cadre de notre thèse. 
Pour conclure, nous reviendrons sur un des présupposés épistémologiques de l'activité versus une épistémologie des savoirs. Nous avons pu observer que le fait de se centrer, en formation, sur des couplages types, peut avoir des incidences sur la transformation de l'activité et sur le sujet lui-même. Ainsi, on peut penser que les dispositifs de formation centrés sur la transformation du sujet (qui intègrent des processus intentionnels, émotionnels, perceptifs et interprétatifs...) ont potentiellement une incidence sur le développement professionnel, sans pour autant tabler sur la base de répertoires de savoirs professionnels à maîtriser. Cette démarcation ne signifie pas qu'il faille pour autant écarter les savoirs disciplinaires de la formation, mais que ces derniers ne constituent pas la porte d'entrée prioritaire de la conception des dispositifs, dans la mesure où on cherche à rendre compte du caractère global du couplage acteur-environnement.

Il faut insister aussi sur la nécessité d'un «cadre participatif» identifié (Goffmann, 1974) pour favoriser la création d'une communauté de pratique au sein d'un collectif. L'impact du collectif dans les transformations du sujet n'a pas été développé ici mais il n'en demeure pas moins un facteur fondamental comme nous avons eu l'occasion de le démontrer dans le cadre de notre thèse ${ }^{22}$.

Ces présupposés semblent tout à fait opératoires dans le cas d'un curriculum par le jeu dramatique, dans lequel on ne parie pas sur un transfert de connaissances du terrain de la formation sur le terrain professionnel (l'enseignant n'est pas un comédien) mais sur une dynamique d'émergence de compétences qui naissent de l'activité théâtrale (le lâcher-prise, la disponibilité à l'environnement), compétences que nous proposons de qualifier d'enactives.

Ainsi, l'évolution de l'engagement de Clara, telle qu'elle apparaît dans la perspective diachronique de cette étude, incite à considérer l'utilisation de la pratique fictionnelle permise par le jeu dramatique comme un vecteur de trajectoire d'apprentissage-développement, propice au développement personnel comme professionnel, propice à l'émancipation par rapport à des représentations réductrices que l'on peut avoir de soi-même.

\section{Références}

Albero, B., (2010). La formation en tant que dispositif : du terme au concept. La technologie de l'éducation : recherches, pratiques et perspectives, 47-59.

Altet M., (2000). L'analyse de pratiques, une démarche de formation professionnalisante ? Revue Recherche et Formation. (35), 25-41.

Archieri, C., (2013). La construction de l'expérience par le théâtre : contribution à un programme d'ergonomie des situations de formation au métier d'enseignant (thèse de doctorat non publiée, Université de Bretagne occidentale-Brest). http://hal.univ-brest.fr/tel-00865641/

Archieri, C., (2013). Pratiques théâtrales et formation aux métiers de l'intervention. Dossier « la formation et le théâtre ». Revue Éducation permanente. (194), 123-134.

Barbier, J.-M., (1996). De l'usage de la notion d'identité en recherche, notamment dans le domaine de la formation. Revue Éducation Permanente. (128), 11-26.

Bourdieu, E., (1998). Savoir faire. Contribution à une théorie dispositionnelle de l'action. Paris : PUF.

Bourgeois, E., (1998). Apprentissage, motivation et engagement en formation. Revue Éducation Permanente. $136(3), 101-109$

\footnotetext{
${ }^{22}$ Cf. http://hal.univ-brest.fr/tel-00865641/
} 
Durand, M., (2008). Un programme de recherche technologique en formation des adultes : une approche enactive de l'activité humaine et l'accompagnement de son apprentissage/développement. Revue Éducation \& Didactique. 2 (3), 97-121.

Goffman, E., (1974). Frame Analysis : An Essay on the Organization of Experience. New York : Harper \& Row. Guérin, J., (2012). Activité collective et apprentissage. De l'ergonomie à l'écologie des situations de formation. Paris : 1'Harmattan.

Guérin, J., \& Archieri, C. (2013). Pratique du jeu dramatique et construction de l'expérience. Recherche \& formation, (2), 75-90.

Jullien, F., (2001). Du « Temps ». Éléments d’une philosophie du vivre. Paris : Grasset.

Jullien, F., (2009). Les transformations silencieuses. Paris : Grasset.

Kaddouri, M., (2000). Enseignant-formateur : la construction de l'identité professionnelle, Recherche et Formation. Paris : L'Harmattan.

Lahire, B., (1998). L'homme pluriel. Les ressorts de l'action. Paris : Nathan.

Leblanc, S. Ria, L., Dieumegard, G., Serres, G. Durand, M. (2008). Concevoir des dispositifs de formation professionnelle des enseignants à partir de l'analyse de l'activité dans une approche enactive. @ctivités, 5 (1), 20-40.

Lemke, J.L., (2000). Material sign processes and ecosocial organization. In P.B. Andersen, C. Emmeche, N.O. Finnemann, \& P.V. Christiansen (Eds.), Downward Causation. Minds, Bodies and Matter, Aarhus : Aarhus University Press, 181-213.

Page, Ch., (2001). Une activité de jeu dramatique dans la formation des futurs enseignants. Communication au colloque de l'AECSE. Toulouse. Revue Connexions. (75), 86-101.

Page, Ch., (2004). Historique du jeu dramatique. Droits réservés.

Peirce, C.S., (1995). Le raisonnement et la logique des choses. Paris : les Éditions du Cerf.

Ria, L., Sève, C., Durand, M., Bertone, S., (2004). Indétermination, contradiction et exploration : trois expériences typiques des enseignants débutants en éducation physique. Revue des Sciences de l'éducation. $30(3), 535-554$.

Ryngaert, J.P., (1991). Le jeu dramatique en milieu scolaire. Bruxelles : De Boeck.

Theureau, J., (2004). L'hypothèse de la cognition (ou action) située et la tradition d'analyse du travail de l'ergonomie de langue française. Revue électronique@ctivités. 11.

Theureau, J., (2004). Le cours d'action : Méthode élémentaire. Toulouse : Octarès.

Theureau, J., (2006). Le cours d'action : Méthode développée. Toulouse : Octarès.

Theureau, J., (2009). Le cours d'action : Méthode réfléchie. Toulouse : Octarès.

Varela, F. (1989). Autonomie et connaissance. Paris : Seuil.

Varela, F.J., (1996). Quel savoir pour l'éthique? Paris : La Découverte.

Vermersch, P. (1994). L'entretien d'explicitation. Paris : ESF.

Vermersch, P., (2002). Conscience réfléchie et recueil de données de verbalisation. Texte présenté aux journées Act'ing de Nouans-le-Fuzelier.

Zeitler, A. (2011). Les apprentissages interprétatifs. Interprétation en action et construction de l'expérience. Paris : L'Harmattan. 


\section{Présentation de l'auteur :}

Catherine Archieri est formatrice à l'ESPE de Bretagne, site de Brest - Université de Bretagne occidentale (UBO) et docteure associée au Centre de Recherche sur l'Éducation les Apprentissages et la Didactique (CREAD - EA 3875), axe Dispositifs, Instruments et Activité (DIA). 